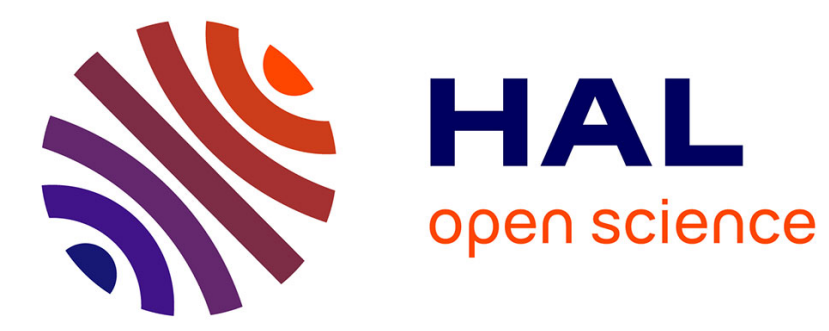

\title{
Optimization of an in vitro chemotherapy to avoid resistant tumours \\ Cécile Carrère
}

\section{To cite this version:}

Cécile Carrère. Optimization of an in vitro chemotherapy to avoid resistant tumours. Journal of Theoretical Biology, 2016, 413, pp.24-33. 10.1016/j.jtbi.2016.11.009 . hal-01302003v2

\section{HAL Id: hal-01302003 \\ https://hal.science/hal-01302003v2}

Submitted on 11 Nov 2016

HAL is a multi-disciplinary open access archive for the deposit and dissemination of scientific research documents, whether they are published or not. The documents may come from teaching and research institutions in France or abroad, or from public or private research centers.
L'archive ouverte pluridisciplinaire HAL, est destinée au dépôt et à la diffusion de documents scientifiques de niveau recherche, publiés ou non, émanant des établissements d'enseignement et de recherche français ou étrangers, des laboratoires publics ou privés.

\section{(ㅇ)(1) $\$$}

Distributed under a Creative Commons Attribution - NonCommercial - NoDerivatives| 4.0 


\title{
Optimization of an in vitro chemotherapy to avoid resistant tumours
}

\author{
Cécile Carrère*
}

November 2, 2016

\begin{abstract}
Chemotherapy use against solid tumours often results in the resistance of the cancer cells to the molecule used. In this paper, we will set up and analyse an ODE model for heterogeneous in vitro tumours, consisting of cells that are sensitive or resistant to a certain drug. We will then use this model to develop different protocols, that aim at reducing the tumour volume while preserving its heterogeneity. These drug administration schedules are determined through analysis of the system dynamics, and optimal control theory.
\end{abstract}

Keywords: Chemotherapy; Mathematical model; Optimal control; Metronomics

\section{Introduction}

Resistance to chemotherapy is a major problem when treating cancer. Diverse factors can be the cause of such a phenomenon. The resistance might be caused by an overall bad adsorption of the drug by the organism: it is possible that the chemical agent is directly evacuated, without even reaching the target cells. This problem is mainly referred as an intrinsic resistance. Another reason is due to the nature of the cancerous cells themselves. Mutations can create new lineages of cells less sensitive to the drug, or an epigenetic behaviour might be selected to tackle the chemical aggression. The way those cells consume nutrients, or divide up, might evolve under the selection pressure imposed by the drug injection. In that case, either the mutation (or the epigenetic behaviour) is present in some cells before the beginning of the treatment, and then selected, or a secondary metabolism way might be chosen to bypass the first one, blocked by the drug action. Either way, when resistance to the chemotherapy appears, the medical team often has no choice but to switch to a different therapy, sometimes more harmful than the first one for the rest of the patient's organism. To prevent such a scenario, different strategies are studied. Anti-angiogenic drugs for example, target endothelial cells, which are less likely to mutate and develop resistance, in order to prevent vascularization of the tumour and maintaining it at a small size [1. Another approach is to change the rhythm of drug injections, as low dose treatments or resting times can have a resensitizing action [13].

In many chemotherapy protocols, the usual procedure is to try to kill as many cancerous cells as possible and very quickly, so that the patient gets rid of his tumour with a maximum probability. Thus a large dose of drug is delivered during very short periods: such a protocol uses the Maximal Tolerated Dose (MTD), which is considered the highest dose of this drug you can give to a patient before side effects are too harmful for him. Such a strategy is justified if

\footnotetext{
*Aix Marseille Université, CNRS, Centrale Marseille, Institut de Mathématique de Marseille, UMR 7373, 13453 Marseille France
} 
the resistance is considered as an acquired trait, and the aim is to destroy the tumour before any mutation occurs. But if resistant cells exist in the tumour even before the chemotherapy begins, then killing all sensitive cells would suppress the only natural control we had on them.

In this outlook, lower and more frequent drug doses that maintain a small sensitive population should be preferred. If a certain amount of them is still present, they might impose a competition pressure on resistant cells, and prevent them from proliferating. Such low dose protocols are called metronomic treatments. By using them, cancer would then be viewed as a long term disease that needs maintenance treatments, because no complete eradication of the disease is considered. They are developed today because they are less harmful for the patient's immune system, and can have an anti-angiogenic effect. Concerning the resistance phenomenon, the doses must be finely tuned to be sure sensitive cells never disappear, while reducing the tumour size to a minimum. This could be done by constant observation of the tumour state, or predicted for example by multiple in vitro experiments, or more efficiently by theoretical and numerical modelling. Moreover, because their advantages over MTD protocols are only effective over a long time, models can help predict the efficiency of such therapies. For both these reasons, mathematical modelling of this competitive environment and analysis of treatment solutions is now crucial. This hindsight can then lead in vitro or in vivo experiments, as it was done in [10].

Treatment optimization has been studied in applied mathematics under different perspectives. Two main trends appear regarding applied analysis and differential equations: numerical optimization for complex systems, or theoretical optimal control of simple systems. In the first category, several drug effects are often taken into account [1]: cytotoxicity, anti-angiogenesis, toxicity for the rest of the organism, action on the immunosystem... Those models might use ODEs to describe a complex system with a lot of interactions [1]. An other form of models is to use replicator dynamics, using individual rules that later infer on the whole system behaviour, as in 20, 2. In that case, numerical methods are used to compute an optimal treatment to reduce, for example, the volume of the tumour under some conditions. But the complexity of such models can hide the driving phenomena of the real system, and they are not practical to predict the general behaviour of the tumour. In the second approach, the model is chosen extremely simple, with few differential equations, so that it can be analytically studied and theoretical optimal control theory can be used. The optimal treatment is determined, or at least characterized by the method given by the Pontryagin Maximum Principle [21]. One can cite [15] for an optimal control of a vascularized tumour through two different drugs given in combination, but also [9, 3 ] for recent applications of optimal control to chemotherapy. This approach gives an insightful comprehension of the model dynamics, and a mathematical justification for the chosen treatment schedule. In order to apply this method to the reduction of a tumour without selecting the resistant cancerous cells, we will work with a simple compartmented ODE cancer model.

In order to gain a better understanding of cancer dynamics, it is now important to see tumours not as homogeneous populations of cells, but as a complex ecosystem with a large variety of phenotypes. When a patient enters a chemotherapy, the medical team chooses to use drugs that target the most common cells in the tumour: this choice divides the cells into two different populations, one that is sensitive to the drug, and one resistant to it. In many cases, this phenotypic difference does not affect the global behaviour of the cells before the chemotherapy. Biological observations are difficult to produce, since it may be hard to differentiate the two lineages. In a series of ongoing experiments, M. Carré at the CRO2 uses fluorescent marking to follow lung cancer cells implantation in Petri wells. A different transmissible marker is implanted to two lineages of cells, one sensitive to Epothilene, coloured in red, and one resistant to it, coloured in green. A first observation is that, when little or no treatment is applied, sensitive cells fill up the well, while resistant cells only survive in a small number, for a large diversity of initial composition. 
Modelling this heterogeneity now depends on the assumptions that are made on the nature of resistant cells. The resistant trait can be considered as continuous, leading to partial differential equations of time and trait [16. In our case, only two kind of cells are taken into account, so ordinary differential equations describing each population dynamics will be used. Random processes occurring in living organisms can be taken into account, like in [17, 8, 12. But since we want to model in vitro experiments, and use the model to develop optimal treatments, we chose a deterministic model. In this line of work, 5, 4] developed simple, linear compartmented models of competing cancer cells, and [14] use optimal control theory on them to determine new treatment schedules that will delay the emergence of a resistance. In these models, the two lineages have the same growth rates, and do not compete for nutrients nor space. This does not explain the dominance of sensitive cells over resistant cells in normal conditions, so further complexifications should be taken into account. A trade-off in growth rate for resistant cells is introduced in [11, so that sensitive cells are selected over them during the experiment, and [18, 19] insist on the role of space and nutrients competition. But in M.Carré's experiments, the dominance of sensitive cells is seen very quickly, even if a first treatment injection has reduced greatly their population at the beginning. Models that do not consider an actual repressive effect of sensitive cells on resistant cells cannot reproduce this phenomenon.

This paper is divided into three main parts. The first part will present a mathematical model for heterogeneous in vitro tumours. Then, after an analysis of the system behaviour under a constant injection of a drug, we investigate a first way to mathematically enhance the treatment protocol, by minimizing the tumour size at the experiment end. This first example being unsatisfactory, the second part studies two other choices of protocols. First, an adaptive stabilization protocol is designed to control indefinitely the system at a low, stable state. Second, we choose to minimize analytically a new cost taking into account the tumour size during the whole experiment. Finally, in the third part, we focus on numerical results, to compare our two protocols with a constant treatment and a cycling MTD protocol.

\section{General study of a heterogeneous in vitro tumour model}

\subsection{Mathematical modelling}

We use phenotypical observations of lung cancer cells A549, sensitive to Epothilene, and their resistant version, A549 Epo40. These lineages are used by M. Carré, researcher at the CRO2 ${ }^{1}$ in experiments on cancerous cells interactions. We also only consider two months in vitro experiments, where only the two types of cells are present, and nutrients are distributed in abundance.

The cell population is divided into two compartments: the sensitive cells, denoted $s$, and the resistant cells, denoted $r$. We neglect any mutation or metabolism change that would make sensitive cells become resistant during the experiment: such events are rare, and would not contribute a lot to the global resistance over two months of treatment. Moreover, the resistant ligneage of cells is resistant to very high concentrations of Epothilene $(40 \mathrm{nMol})$, while dosages in this kind of experiment is limited to tolerable levels for an animal organism ( $5 \mathrm{nMol})$. Hence the selective pressure is not sufficient to make A549 cells mutate into A549 Epo40 cells. We thus decided to focus on other dynamics that explain the emergence of a globally resistant tumour. When cultivated alone, the cells behave as in a logistic model, which we will use for the rest of the study. This model is expressed for a generic population $x$ as:

$$
\frac{d x}{d t}(t)=\rho x(t)\left(1-\frac{x(t)}{K}\right)
$$

\footnotetext{
${ }^{1}$ Center for Research in Oncobiology and Oncopharmacology
} 
where $\rho$ is the initial growth rate of the population, and $K$ the carrying capacity of the environment. The term $\frac{x}{K}$ represents the total space already occupied in the environment. In our case, the two kinds of cells consume space, so this term should be $\frac{s+r}{K}$. But we should take into account the difference of size between the two lineages: resistant cells are bigger than sensitive cells in M. Carré's experimental setting. For that purpose, the term representing the used space is $\frac{s(t)+m r(t)}{K}$, with $m>1$. The growth rates of $s$ and $r$ are denoted, respectively, $\rho_{s}$ and $\rho_{r}$.

$$
\left\{\begin{array}{l}
\frac{d s}{d t}(t)=\rho_{s} s\left(1-\frac{s+m r}{K}\right) \\
\frac{d r}{d t}(t)=\rho_{r} r\left(1-\frac{s+m r}{K}\right) .
\end{array}\right.
$$

When cultivated together without any treatment, A549 cells will outgrow the A549Epo40 population quickly. But in the framework of a logistic model, (1) is not sufficient to describe this phenomenon, as the quantity $\rho_{r} \ln (s(t))-\rho_{s} \ln (r(t))$ here remains constant. Moreover, when cultivated separately, the two lineages seem to have very similar growth coefficients $\rho_{s}$ and $\rho_{r}$ (see TAB 1). Thus, we add a supplementary competition term $-\beta s r$ for the resistant cells. This ensures that the tumour will be, after some time, composed of almost only sensitive cells. It is coherent with our idea of a resistance that cannot be detected before treatment, as very few cells present this trait.

Finally, the concentration $C(t)$ of the drug only acts on the sensitive cells, and its action is proportional to the number of those cells. We restrict our model to the case $0 \leq C(t) \leq C_{\max }$, where $C_{\max }$ is the maximal tolerated dose for a patient (MTD). Resistant cells A549Epo40, for this range of concentration, are completely unaffected by the drug. The system is now:

$$
\left\{\begin{array}{l}
\frac{d s}{d t}(t)=\rho_{s} s\left(1-\frac{s+m r}{K}\right)-\alpha s C(t), \\
\frac{d r}{d t}(t)=\rho_{r} r\left(1-\frac{s+m r}{K}\right)-\beta s r .
\end{array}\right.
$$

The different parameters in ТАB.1 are estimated values for A549 (sensitive) and A549 Epo40 (resistant) cells.

Theorem 1. For any bounded Lebesgue measurable function $C:[0, T] \rightarrow\left[0, C_{\max }\right]$ with $T \leq \infty$, the triangle $\mathbb{T}:=\{s>0\} \cap\{r>0\} \cap\{s+m r<K\}$ is positively invariant under the dynamical system of equations 2 .

Proof. A direct application of theorem 2.1.1. in [7. p. 14] ensures that for any $\left(s_{0}, r_{0}\right) \in \mathbb{T}$ and measurable control $C:[0, T] \rightarrow\left[0, C_{\max }\right]$, the initial value problem (2) with $(s, r)(0)=\left(s_{0}, r_{0}\right)$ has a global unique solution $(s, r):[0, T] \rightarrow \mathbb{R}^{2}$, which is absolutely continuous on $[0, T]$ and depends continuously on the initial condition.

Let $\{(s, r)(t), t \in[0, T]\}$ be a trajectory of the system (2) for a certain Lebesgue measurable function $C:[0, T] \rightarrow\left[0, C_{\max }\right]$ and such that $(s, r)(0)=\left(s_{0}, r_{0}\right)$.

On the set $\{s=0\}$ (resp. $\{r=0\}$ ), the system (2) yields $\frac{d s}{d t}=0$ (resp. $\frac{d r}{d t}=0$ ). So according to the uniqueness of the solution $(s, r)$, if there exists a certain $t_{0}$ such that $s\left(t_{0}\right)=0$ (resp. $\left.r\left(t_{0}\right)=0\right)$, then for all $t \in[0, T], s(t)=0(\operatorname{resp} r(t)=0)$. Moreover, the points $O=(0,0)$ and $E_{r}=\left(0, \frac{K}{m}\right)$ are fixed points, and at the point $K=(K, 0)$ the derivative on $s$ satisfies $\frac{d s}{d t} \leq 0$. Hence the sets $\left\{s=0,0 \leq r \leq \frac{K}{m}\right\}$ and $\{0 \leq s \leq K, r=0\}$ are both stable under the system (2).

Now suppose a trajectory $\{(s, r)(t), t \in[0, T]\}$ satisfies $(s, r)(0) \in \mathbb{T}$, and there exists $t>0$ such that $(s, r)(t) \notin \mathbb{T}$. Then by continuity there exists a smallest time $t_{0}>0$ such that $(s, r)\left(t_{0}\right) \in \partial \mathbb{T}$. The cases were $s\left(t_{0}\right)=0$ or $r\left(t_{0}\right)=0$ are ruled out by the stability of the sets $\{s=0\}$ and $\{r=0\}$. Hence the only possibility is $s\left(t_{0}\right)+m r\left(t_{0}\right)=K$ with $s\left(t_{0}\right)>0$ and $r\left(t_{0}\right)>0$. But then $\frac{d s}{d t}\left(t_{0}\right)+m \frac{d r}{d t}\left(t_{0}\right)=-\alpha s\left(t_{0}\right) C\left(t_{0}\right)-\beta s\left(t_{0}\right) r\left(t_{0}\right)<0$, so there exists $\epsilon>0$ 


\begin{tabular}{|c|c|c|c|}
\hline Symbol & Meaning & Value & Unit \\
\hline$s_{0}$ & sensitive cells number & initial $: 5000$ & cells \\
$r_{0}$ & resistant cells number & initial $: 5000$ & cells \\
$K$ & Petry dish carrying capacity & 4800000 & cells \\
$m$ & size ratio between $s$ and $r$ & 30 & adimensional \\
$\rho_{s}$ & sensitive cells growth rate & 0.031 & cells $/$ hour \\
$\rho_{r}$ & resistant cells growth rate & 0.026 & cells $/$ hour \\
$C$ & drug concentration & maximum $: 5$ & $\mathrm{nM} / \mathrm{hour}^{-1}$ \\
$\alpha$ & drug effect & 0.06 & $\mathrm{nM} \mathrm{Cols}^{-1}$ \\
$\beta$ & action of sensitive on resistant & $6.25 \cdot 10^{-7}$ & cells \\
$T$ & experiments duration & 720 & $\mathrm{~h}$ \\
\hline
\end{tabular}

Table 1: Parameters for the equations and their value used in the simulations

such that $s\left(t_{0}-\epsilon\right)+m r\left(t_{0}-\epsilon\right)>K$. This is absurd since $t_{0}$ was the first exiting time by definition.

Hence $\mathbb{T}$ is positively stable under system (2).

In equations (2), the two growth rates are taken different. For the lineages we consider, they are quite similar (see TAB.1). Thus in the rest of this article we consider $\rho_{s}=\rho_{r}=$ $\rho=0.03$ cells/hour. This will simplify the computations, but the results remain similar if the difference is kept. The treatment effect could also be considered as a saturated function, with $\alpha C$ replaced by $\frac{\alpha C(t)}{E+C(t)}$, but this does not change much the different results we obtained, so we decided to keep a simple model.

In the end, the system we will study is:

$$
\left\{\begin{array}{l}
\frac{d s}{d t}(t)=\rho s\left(1-\frac{s+m r}{K}\right)-\alpha s C(t) \\
\frac{d r}{d t}(t)=\rho r\left(1-\frac{s+m r}{K}\right)-\beta s r \\
s(0)=s_{0} \quad, \quad r(0)=r_{0}
\end{array}\right.
$$

\subsection{Phase plan analysis for a constant treatment}

In order to understand the system (3) dynamic, we first study its behaviour under a constant treatment over time $C(t)=C$

$$
\left\{\begin{array}{l}
\frac{d s}{d t}(t)=\rho s\left(1-\frac{s+m r}{K}\right)-\alpha s C, \\
\frac{d r}{d t}(t)=\rho r\left(1-\frac{s+m r}{K}\right)-\beta s r .
\end{array}\right.
$$

This study will highlight the global behaviour of the system, and show important points in the phase plan. Under this assumption, for a constant treatment, we have the following theorem:

Theorem 2. Given an initial condition $\left(s_{0}, r_{0}\right) \in \mathbb{T}$, the system (4) will evolve to different values depending on the treatment value. Denoting $C_{\text {metro }}:=\frac{K \beta}{\rho+K \beta} \frac{\rho}{\alpha}$, we have:

- if $C=0$ then $(s, r)(t) \underset{t \rightarrow \infty}{\longrightarrow}(K, 0)$,

- if $0<C<C_{\text {metro }}$ then, depending on $\left(s_{0}, r_{0}\right)$, either $(s, r)(t) \underset{t \rightarrow \infty}{\longrightarrow}\left(K \frac{\rho-\alpha C}{\rho}, 0\right)$ or $(s, r)(t) \underset{t \rightarrow \infty}{\longrightarrow}$ $\left(0, \frac{K}{m}\right)$, 
- if $C \geq C_{\text {metro }}$ then $(s, r)(t) \underset{t \rightarrow \infty}{\longrightarrow}\left(0, \frac{K}{m}\right)$.

Proof. A study of the system (4) fixed points and their stability, depending on the value of $C$, shows that three different cases emerge. They are summed up on Fig.1

\section{- Case 1: No treatment}

If no treatment is applied, then the only attractive fixed point of the system is $E_{s}(0):=$ $(K, 0)$, where the well is completely filled and with only sensitive cells. All trajectories starting in $\mathbb{T}$ are attracted to it, as illustrated on FIG 1 a.

\section{- Case 2: Strong treatment}

If a strong treatment is injected, namely $C \geq C_{\text {metro }}:=\frac{K \beta}{\rho+K \beta} \frac{\rho}{\alpha}$, then the only attractive fixed point is $E_{r}:=\left(0, \frac{K}{m}\right)$, where the well is filled with only resistant cells at their maximal stacking capacity. All trajectories starting inside $\mathbb{T}$ are attracted to this point, as illustrated in FIG, 1C,

\section{- Case 3: Weak treatment}

In the case where $0<C<C_{\text {metro }}$, two fixed points are now stable: $E_{r}=\left(0, \frac{K}{m}\right)$ with only resistant cells, and $E_{s}(C):=\left(K \frac{\rho-\alpha C}{\rho}, 0\right)$, with only sensitive cells, but at a lower level than if no treatment is used. Depending on the initial number of both sensitive and resistant cells, trajectories will evolve towards one or the other of the fixed points. The triangle $\mathbb{T}$ is thus divided into two attraction basins, corresponding to $E_{s}(C)$ and $E_{r}$.

In each case, Poincaré-Bendixson theorem allows us to conclude.

A third fixed point exists, which is always unstable: $E_{u}(C):=\left(s_{u}(C), r_{u}(C)\right)$ with $s_{u}(C):=$ $\frac{\alpha C}{\beta}$ and $r_{u}(C)=\frac{\rho+K \beta}{m \beta \rho}\left(\frac{\rho K \beta}{\rho+K \beta}-\alpha C\right)$, a mixed population state. Numerically, we can estimate that the attraction basins delimitation is a convex curve going through $O:=(0,0)$ and $E_{u}(C)$. In any case, one sees that if at a certain time $t$ we have $s(t)>s_{u}(C)$ and $r(t)=r_{u}(C)$, then $\frac{d r}{d t}(t)<0$, and if $s(t)=s_{u}(C)$ and $r(t)<r_{u}(C)$ then $\frac{d s}{d t}(t)>0$. Hence the plane portion $\left\{s>s_{u}(C)\right\} \cap\left\{r<r_{u}(C)\right\}$ is positively stable, so it is contained into $E_{s}(C)$ attraction basin.

When $C$ approaches the limit value $C_{\text {metro }}$, the point $E_{s}(C)$ approaches its limit value $E_{s, l}:=$ $\left(\frac{K \rho}{\rho+K \beta}, 0\right)$. It is an interesting value, as no smaller population with only sensitive cells is stable.

FIG 1 b represents the situation of a weak treatment.

With values from ТАВ.1 we find that $C_{\text {metro }}=0.495 n M / h$. This corresponds to a coherent value for metronomic treatments, that use a dose ten times weaker than the MTD in classical protocols.

\subsection{Optimal control of the tumour size at the end of the experiment}

Our model is able to reproduce the phenomena we desired. It now should be used to determine treatments that achieve medical purposes. The choice of an objective, mathematically, will greatly influence the form of the corresponding optimal treatment. Thus, we investigate different kinds of objectives to present their advantages and drawbacks. We present here a first example, to reduce the tumour size at the end of the experiment. 


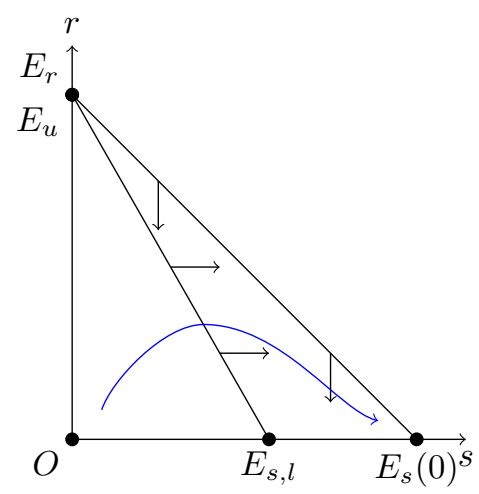

(a) No treatment

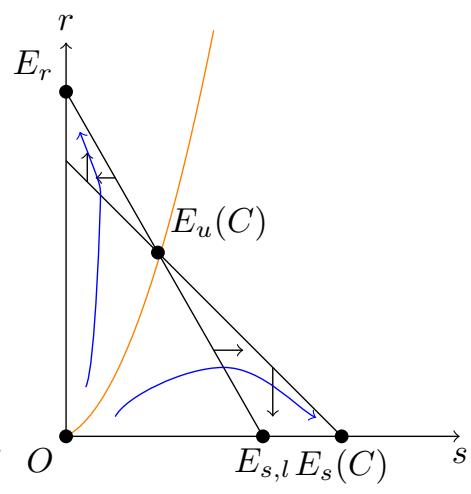

(b) Weak treatment

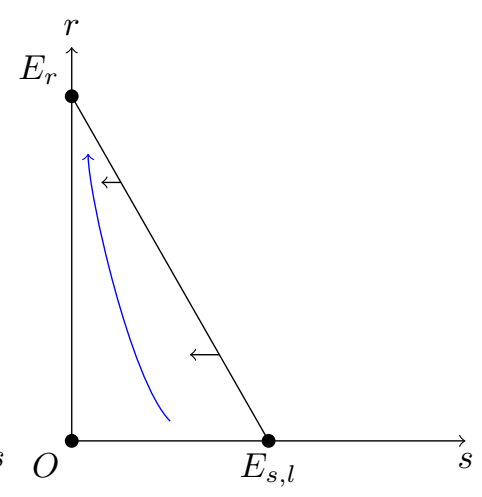

(c) Strong treatment

Figure 1: Phase planes for the three different situations, the model is able to reproduce the results from experiments

Optimal Control Problem 1. Given an initial condition $\left(s_{0}, r_{0}\right) \in \mathbb{T}$, a maximal concentration of the treatment $C_{\max }$ and a duration $T$ of experiment, find a Lebesgue measurable function $C:[0, T] \rightarrow\left[0, C_{\max }\right]$ that minimizes the global quantity of effective cancerous cells:

$$
\psi(s, r):=s(T)+m r(T)
$$

at the final time $T$.

This is a Mayer problem [21]. The answer of this problem highlights how it is not relevant for medical purposes:

Theorem 3. The optimal treatment for Problem 1 is "bang-bang", and up to a certain time $t_{\text {start }} \in[0, T]$ depending on the parameters, we have $C(t)=0$, and from $t_{\text {start }}$ to $T$ the treatment is maximal $C(t)=C_{\max }$.

Proof. We should first state the existence of optimal controls solution to Problem 1 We will use Theorem 5.1.1. from [7, p. 88]. We denote $f:((s, r), t, C) \mapsto\left(\rho s\left(1-\frac{s+m r}{K}\right)-\alpha s C, \rho r(1-\right.$ $\left.\left.\frac{s+m r}{K}\right)-\beta s r\right)$. First, for any $t \in[0, T]$ and any $(s, r) \in \mathbb{T}$, the set of velocities $F((s, r), t)=$ $\left\{f((s, r), t, C), C \in\left[0, C_{\max }\right]\right\}$ is convex. Second, $f$ is continuous in all variables and differentiable w.r.t. $(s, r)$. Third, by cut-off far from $\mathbb{T}$, we can make so that for all $(s, r), t, C$ we have $|f((s, r), t, C)| \leq \tilde{C}(1+|(s, r)|)$ where $\tilde{C}$ is a constant depending on $f$ and our cut-off choice. All the assumptions of the theorem are satisfied, thus there exists an optimal solution to Problem 1 .

Now we will characterize such an optimal solution. The necessary conditions for the optimality of the control $C:[0, T] \rightarrow\left[0, C_{\max }\right]$ of the Pontryagin Minumum principle state that there exist a constant $p_{0} \geq 0$ and an absolutely continuous adjoint vector $p:[0, T] \rightarrow \mathbb{R}^{2}$ satisfying the following conditions. First, for all $t \in[0, T]$ we have $\left(p_{0}, p(t)\right) \neq 0$, that is the condition of non-triviality. Second, the optimality condition: the optimal control $C^{*}(t)$ minimizes along the optimal trajectory $\left(p_{0}, p(t), s^{*}(t), r^{*}(t)\right)$ the Hamiltonian:

$$
H\left(s, r, p_{1}, p_{2}, C\right):=p_{1} \cdot\left(\rho s\left(1-\frac{s+m r}{K}\right)-\alpha s C\right)+p_{2} \cdot\left(\rho r\left(1-\frac{s+m r}{K}\right)-\beta s r\right),
$$




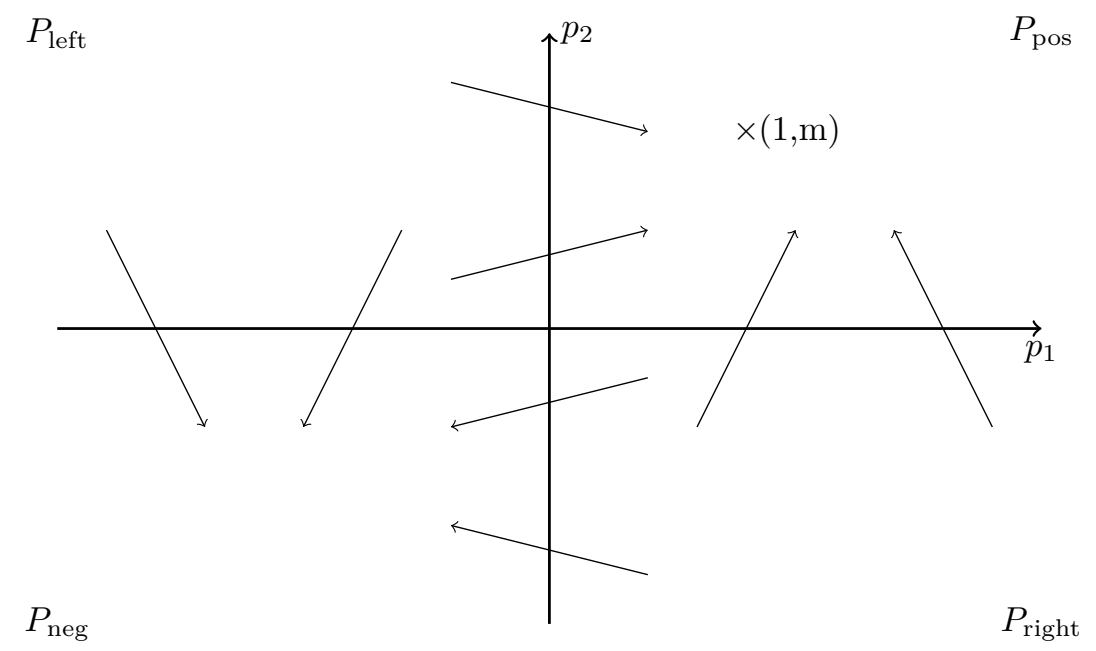

Figure 2: The phase plane of the adjoint vector $p$ reveals two positively stable zones

over the set $\left[0, C_{\max }\right]$. Third, $p$ satisfies the adjoint equations and transversality condition:

$$
\left\{\begin{array}{l}
\dot{p}(t)=-\left(\begin{array}{l}
\frac{\partial H}{\partial s} \\
\frac{\partial H}{\partial r}
\end{array}\right)(t)=-\left(\begin{array}{cc}
\rho\left(1-\frac{2 s+m r}{K}\right)-\alpha C & -\left(\frac{\rho}{K}+\beta\right) r \\
-\rho s \frac{m}{K} & \rho\left(1-\frac{s+2 m r}{K}\right)-\beta s
\end{array}\right) p(t), \\
p(T)=\nabla \psi(s(T), r(T))=p_{0}\left(\begin{array}{c}
1 \\
m
\end{array}\right) .
\end{array}\right.
$$

If $p_{0}=0$, then $(6)$ implies that $p_{1}(T)=p_{2}(T)=0$ : this contradicts the non-triviality condition, so $p_{0} \neq 0$. Hence we can normalize $p_{0}=1$ without loss of generality.

The hamiltonian $H$ is linear in the control $C$ : the function $-\alpha p_{1} s$ is called the switching function of the problem. In order to minimize $H$ along the optimal trajectory, we see that:

- if $\alpha p_{1}^{*}(t) s^{*}(t)<0$ then $C^{*}(t)=0$,

- if $\alpha p_{1}^{*}(t) s^{*}(t)>0$ then $C^{*}(t)=C_{\max }$,

- if $\alpha p_{1}^{*}(t) s^{*}(t)=0$ we need more information.

Since $\frac{d p_{1}^{*}}{d t}(t)=-\left(\rho\left(1-\frac{2 s^{*}+m r^{*}}{K}\right)-\alpha C^{*}(t)\right) p_{1}^{*}(t)+\left(\frac{\rho}{K}+\beta\right) p_{2}^{*}(t)$, we have $p_{1}^{*}(t) \equiv 0$ on a non empty interval $I$ if and only if $p_{2}^{*}(t) \equiv 0$ on that same interval. But then according to the Cauchy-Lipschitz theorem $I=(0, T)$, so $p(T)=0$. This is absurd, so $p_{1}^{*}$ does not vanish on an interval. Hence the optimal treatment is for every $t \in[0, T]$ either 0 or $C_{\max }$.

A study of $p$ phase plan FIG. 2 shows that any trajectory ending on $\left(\begin{array}{c}1 \\ m\end{array}\right)$ either satisfies $p_{1}(t)>0$ for every $t>0$, or there exists $t_{0}>0$ such that $p_{1}(t)<0$ for every $0<t<t_{0}$ and $p_{1}(t)>0$ for every $t>t_{0}$.

If $p_{1}^{*}(0)>0$ then $p_{1}^{*}>0$ during the whole experiment, so $\forall t \in[0, T], C(t)=C_{\max }$, so $t_{\text {start }}=0$. If $p_{1}^{*}(0)<0$ then $\forall t \in\left[0, t_{0}\right), p_{1}^{*}(t)<0$ and $\forall t \in\left(t_{0}, T\right], p_{1}^{*}(t)>0$, so with $t_{\text {start }}=t_{0}$ we have $\forall t \in\left[0, t_{\text {start }}\right), C(t)=0$, and $\forall t \in\left(t_{\text {start }}, T\right], C(t)=C_{\max }$. 


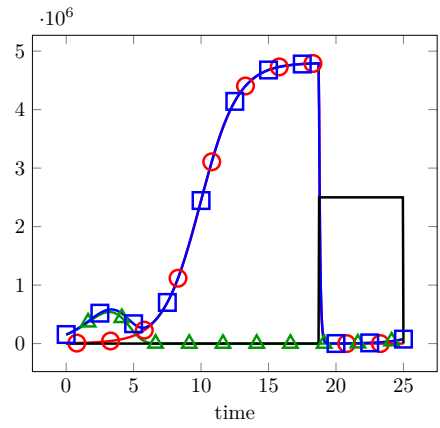

(a) Optimal treatment is applied for the time of the experiment $T=20$, killing most sensitive cells at the end

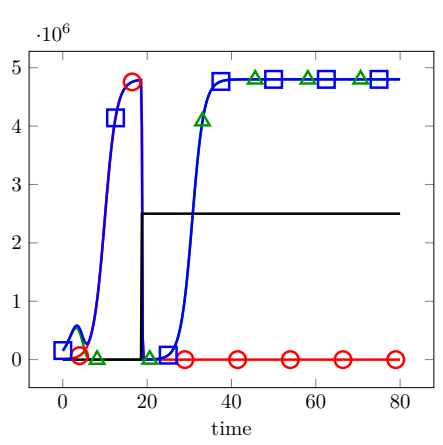

(b) If the treatment continues after $T$, resistant cells invade the well at their maximal level

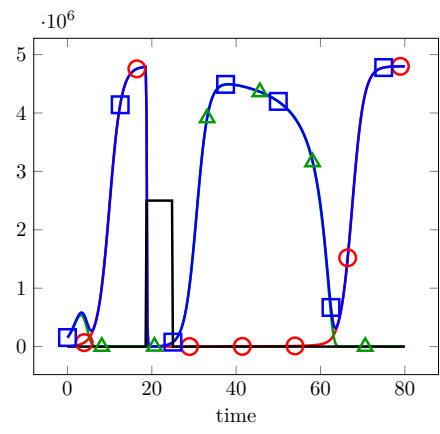

(c) If we end the treatment after $T$, sensitive cells regain strength and resettle the well after some recovery time

$\checkmark$ sensitive $s \triangle$ effective resistant $m r$ 曰 effective total $s+m r-$ treatment

Figure 3: Cells populations evolutions during an optimal treatment when only the final population is take into account

In other words, we wait for the sensitive cells to invade the dish and kill almost all resistant cells, then just before the measurement time we release a huge dose of drugs to clear off the sensitive cells. The treatment starting time $t_{\text {start }}$ can be determined numerically.

This is not an acceptable strategy for clinical research: indeed, it supposes that we only want a pointwise result, without looking at the intermediate states of the system, nor the long term consequences of such a protocol. Indeed, it is possible that we let the tumour reach its maximal capacity $K$ during the experiment, as seen in FIG.3a. which would be really bad in a medical context. Moreover, according to our model, if after the end of the experiment $T$ one maintains the treatment $C=C_{\max }$, then the resistant cells will invade the now free space left over by the sensitive cells as seen on Fig. $3 \mathrm{~b}$. Otherwise, if one stops the treatment after $T$, so $C=0$, then the sensitive cells will progressively resettle in the well as seen on FiG. $3 \mathrm{c}$. Even worse, such a large drug dose might have killed, in reality, all the sensitive cells: even if we stop the treatment after $T$, we are no longer able to control the tumour. To tackle this problem, new treatment protocols should take into account the quantity of cells in the well during the whole experiment, from 0 to $T$, and have an outlook on future behaviour on the solution.

This example highlighted that the choice of a cost $\psi$ is very important to define an optimal therapy. It depends on medical objectives, and should be chosen after discussions with biologists.

\section{Treatment protocols with different aims}

Since the optimal treatment defined in 2.3 is not very well suited for medical protocols, we define two new problems. The first one based solely on the study of trajectories in 2.2 gives a way to stabilize the tumour at low, constant state. The second one is a new optimal control problem, which takes into account the size of the tumour during the whole experiment. 


\subsection{Adaptive stabilization protocol}

A consequence of Theorem 2 is that the total tumour $s+r$ never goes extinct. For medical reasons, it is interesting to reach a constant state, with mostly sensitive cells, and at the lowest possible level. If we define a treatment so that the tumour reaches such a state, the cancer will no longer be considered a single-event disease, but a chronic one, that requires constant care but is under medical control.

In this outlook, we want to stabilize the system at the fixed point with only sensitive cells $E_{s}(C)$, with this point as close as possible to $O=(0,0)$, with the constraint of $E_{s}(C)$ being

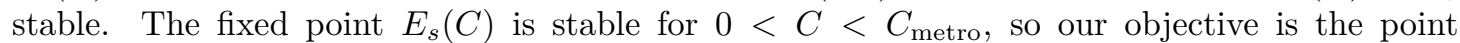
$E_{s, l}:=\left(\frac{K}{\rho+K \beta}, 0\right)$, that is the position of $E_{s}(C)$ for $C=C_{\text {metro. }}$.

We now propose a strategy to approach this point. We want to define a treatment for our system such that, at any moment $t$, the trajectory of the system $(s, r)$ is in the basin of attraction of $E_{s}(C)$, which position depends on the value of $C(t)$. Thus, one can assure the patient that his tumour is controlled. We also impose $C(t)$ to be piecewise constant, and its value can only be changed each day (which corresponds to an experimental situation). The initial population is supposed to be a mix of sensitive and resistant cells $(s(0)>0, r(0)>0)$. This strategy is an adaptive, iterative medical protocol.

The beginning of the $i$ th day is denoted $t_{i}$, and the value of $C$ during the $i$ th day is denoted $C_{i}$. We use a coefficient $\lambda_{i} \in(0,1)$ that is also modified each day. Measures of $(s, r)\left(t_{i}\right)$ define the treatment value each day:

- Initialization $(s, r)(0)$ cells are implanted, $\lambda_{0}=1 / 2$

- Loop for $i=1$ to end of experiment

1. If $r\left(t_{i}\right)<\frac{K}{m}\left(1-\frac{s\left(t_{i}\right)}{K}\right)$, let the system evolve with no treatment, $C_{i}=0$, and $\lambda_{i}=\frac{1}{2}$ for reinitialization.

2. If $r\left(t_{i}\right) \geq \frac{K}{m}\left(1-\frac{s\left(t_{i}\right)}{K}\right)$, set $C_{i}=\lambda_{i} C_{\text {metro }}\left(1-\frac{m}{K} r\left(t_{i}\right)\right)$ and $\lambda_{i+1}=\frac{1+\lambda_{i}}{2}$.

- End of experiment

This protocol is illustrated by FIG,4 during four days: FIG 4 a shows the action of a first step of the protocol, without treatment, while FIG.4b and 4c represent two days with treatment. During the loop, theoretically, phase 1 can only be encountered for a few days at the experiment beginning, and when phase 2 begins it lasts until the end of our protocol. However, biological variations could occur and bring us back to phase 1 .

We have presented the general idea of an algorithm reaching the plateau $(s, r)=E_{s, l}=$ $\left(\frac{K}{\rho+K \beta}, 0\right)$. As shown on FIG 5 the sensitive population reaches a maximal level shortly after the treatment started, then slowly decreases to reach asymptotically the point $E_{s, l}$. The different parameters (time delay before starting the treatment, pace of changes in $C, \lambda$ parameter...) could be optimized by the choice of $\lambda_{i}$, depending on the medical constraints (rhythm of the patient presence at the hospital, toxicity of the treatment) and objectives: reaching the plateau as soon as possible, using the less treatment point-wise or cumulated...

In the following, this protocol will be referred to as the adaptive protocol. It treats the tumour as a chronic disease: we do not try to suppress the cancerous cells, but to control them for an indefinite time. 

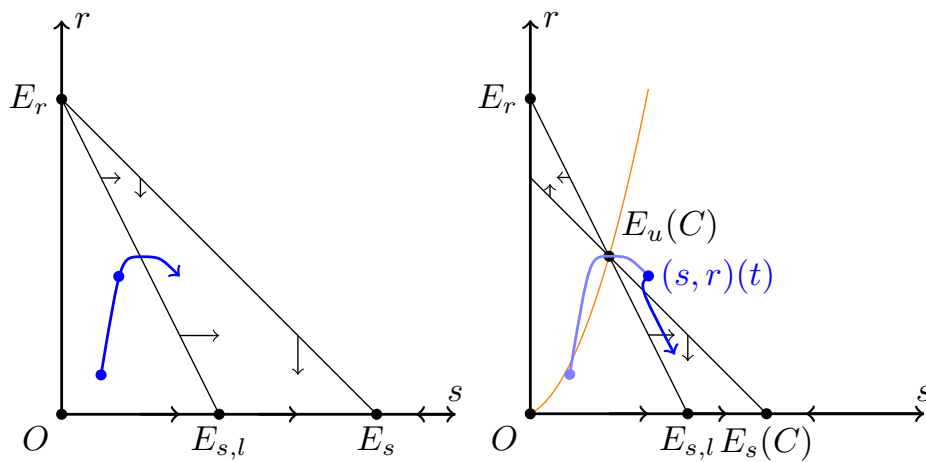

(a) Day 1 and 2: the quantity of resistant cells is still growing, so no treatment is applied (b) Day 3: the system has crossed the designated threshold to start the treatment

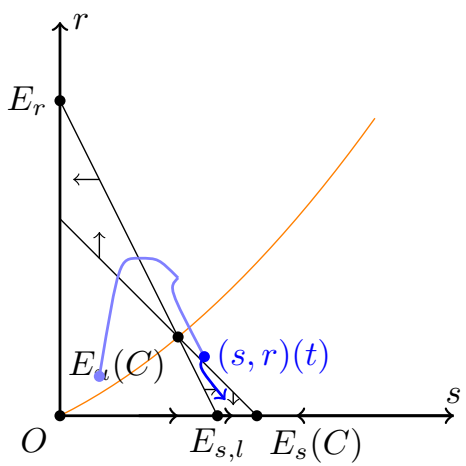

(c) Day 4: the treatment value is actualized to bring $E_{s}(C)$ closer to $E_{s, l}$

Figure 4: Several steps of the adaptive protocol are represented on the phase plane

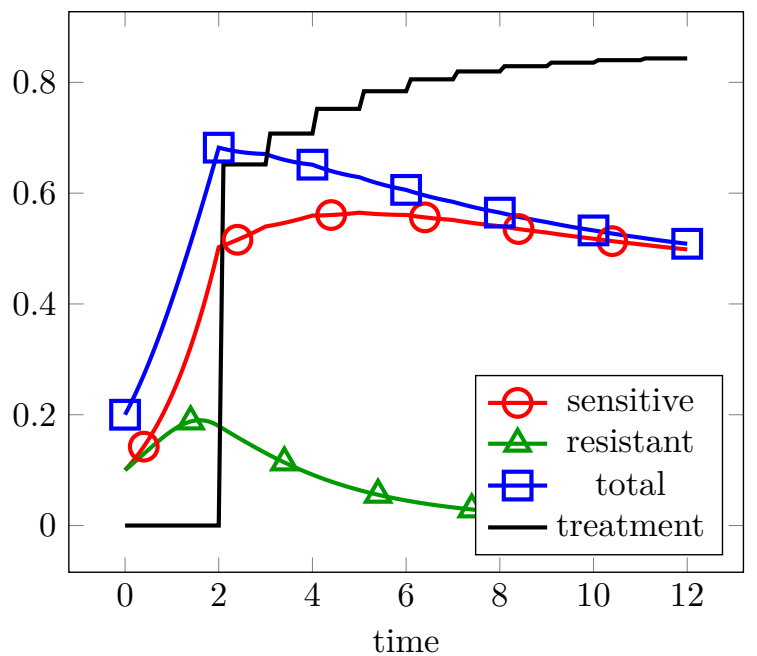

Figure 5: Adaptive stabilization protocol in the time scale: the treatment is increased each day, and the cells population approaches the desired level 


\subsection{Optimal control of the tumour size during the whole experiment}

The adaptive protocol is designed through qualitative considerations on the system behaviour. We now want to determine another type of protocol, that will have a quantitative objective.

A natural way to take into account the treatment effects for an entire period is to use an integral cost: $L_{\text {int }}(T)=\int_{0}^{T}(A s(t)+B r(t)) d t$. For medical reasons, it is also important that during the experiment, the tumour remains small. To ensure that, we will use a cost that penalizes high values of $s(t)$ and $r(t)$, i.e a $L^{2}$-norm: $\int_{0}^{T}\left(A s^{2}(t)+B r^{2}(t)\right) d t$. Our new problem is the following:

Optimal Control Problem 2. Given an initial condition $\left(s_{0}, r_{0}\right) \in \mathbb{T}$, a maximal concentration of the treatment $C_{\max }$ and a time $T$ of experiment, find a piecewise continuous function $C$ : $[0, T] \rightarrow\left[0, C_{\max }\right]$ that minimizes the following cost:

$$
L_{2}(T):=\tilde{\psi}(s(T), r(T))+\int_{0}^{T} L^{0}(s(t), r(t)) d t,
$$

with $\tilde{\psi}$ the Mayer part of the cost, and $L^{0}$ the Lagrange part of it:

$$
\tilde{\psi}(s, r):=s^{2}+r^{2}, \quad L^{0}(s, r):=A s^{2}+B r^{2} .
$$

We find that the optimal treatment for this problem is of a particular form:

Theorem 4. The optimal treatment for Problem 2 is a piecewise continuous function $C$, with three possible values at each time point. Either:

- the control is maximal $C=C_{\max }$,

- the control is minimal $C=0$,

- the control is singular $C=\frac{1}{\alpha s}\left(\frac{B}{A} r^{2}\left(\frac{\rho}{K}+\beta\right)+s \rho\left(1-\frac{s+2 m r}{K}\right)\right)$.

Moreover, the control is maximal at the end of the experiment: $C(T)=C_{\max }$.

Proof. First, the existence of optimal solutions to Problem 2 is ensured by Theorem 5.2.1 from [7, on p.94. The assumptions of this theorem are satisfied by our system inside $\mathbb{T}$, so a cut-off far from it is enough to apply the theorem.

We will once again use the framework of Optimal control theory, and in particular the Pontryagin Minimum Principle. Because the cost functional now has an Lagrangian part, the system hamiltonian is modified:

$$
H\left(s, r, p_{1}, p_{2}, C\right)=A s^{2}+B r^{2}+p_{1} \cdot\left(\rho s\left(1-\frac{s+m r}{K}\right)-\alpha s C\right)+p_{2} \cdot\left(\rho r\left(1-\frac{s+m r}{K}\right)-\beta s r\right)
$$

From this we deduce the differential equations on the adjoint vector $p=\left(\begin{array}{l}p_{1} \\ p_{2}\end{array}\right)$

$$
\left\{\begin{array}{l}
\frac{d p_{1}}{d t}=-\frac{\partial H}{\partial s}=-2 A s-p_{1}\left(\rho\left(1-\frac{2 s+m r}{K}\right)-\alpha C\right)+p_{2}\left(\frac{\rho}{K}+\beta\right) r \\
\frac{d p_{2}}{d t}=-\frac{\partial H}{\partial r}=-2 B r+p_{1} \rho \frac{m}{K} s-p_{2}\left(\rho\left(1-\frac{s+2 m r}{K}\right)-\beta s\right) .
\end{array}\right.
$$

In (7), the treatment $C$ only appears as $-\alpha s p_{1} C$ : the switch function is once again $-\alpha s p_{1}$. From the Pontryagin Minimum Principle we know that: 
- if $\alpha s p_{1}>0$ then $C=C_{\max }$,

- if $\alpha s p_{1}<0$ then $C=0$,

- if $\alpha s p_{1}=0$ then $C$ is to be determined.

Let us now investigate the case $p_{1}(t) \equiv 0$ on $I$, where $I$ is an interval of non-empty interior. In optimal control theory, such an interval is called a singular arc. We want to deduce an expression for $C$ on that interval. First consider (8) on that particular interval:

$$
0=-2 A s+p_{2}\left(\frac{\rho}{K}+\beta\right) r
$$

Differentiating this expression gives us:

$$
\begin{aligned}
\dot{p}_{2}\left(\frac{\rho}{K}+\beta\right) r & =2 A\left(\rho s\left(1-\frac{s+m r}{K}\right)-\alpha s C\right)-p_{2}\left(\frac{\rho}{K}+\beta\right)\left(\rho r\left(1-\frac{s+m r}{K}\right)-\beta s r\right) \\
& =2 A\left(\rho s\left(1-\frac{s+m r}{K}\right)-\alpha s C\right)-2 A s\left(\rho\left(1-\frac{s+m r}{K}\right)-\beta s\right) \\
& =2 A(\beta s-\alpha C) s .
\end{aligned}
$$

Moreover, injecting (10) in 99:

$$
\dot{p}_{2}\left(\frac{\rho}{K}+\beta\right) r=-2 B r^{2}\left(\frac{\rho}{K}+\beta\right)-2 A s\left(\rho\left(1-\frac{s+2 m r}{K}\right)-\beta s\right) .
$$

So that in the end:

$$
(\beta s-\alpha C) s=-\frac{B}{A} r^{2}\left(\frac{\rho}{K}+\beta\right)-s\left(\rho\left(1-\frac{s+2 m r}{K}\right)-\beta s\right) .
$$

We now have an expression for $C$ on a singular arc :

$$
\begin{aligned}
C(t) & =\frac{1}{\alpha s}\left(\frac{B}{A} r^{2}\left(\frac{\rho}{K}+\beta\right)+s \rho\left(1-\frac{s+2 m r}{K}\right)\right)(t) \\
& =\frac{1}{\alpha s}\left(r\left(\frac{B}{A}\left(\frac{\rho}{K}+\beta\right) r-s \rho \frac{m}{K}\right)+s \rho\left(1-\frac{s+m r}{K}\right)\right)(t) .
\end{aligned}
$$

One should note that this can only hold if $0 \leq C \leq C_{\max }$.

Moreover, if we denote $\tilde{\psi}(t)=s^{2}(t)+r^{2}(t)$, such that $\psi(T)=\tilde{\psi}(T)+\int_{0}^{T}\left(A s^{2}(t)+B r^{2}(t)\right) d t$, the Pontryagin Minimum Principle states that the adjoint $p$ corresponding to the optimal control satisfies $p(T)=\nabla \tilde{\psi}(T)$, so $p_{1}(T)=2 s(T)>0$. Hence we have $C(T)=C_{\max }$.

Theorem 4 only states the possible forms of an optimal treatment. Depending on the parameters in the equations, the time $T$ and the initial condition, it might happen that the optimal treatment does not provide any singular arc (or minimal). The second order condition of Legendre-Clebsch [6] states that, on a singular arc,

$$
\frac{\partial}{\partial C} \frac{d^{2}}{d t^{2}} \frac{\partial H}{\partial C}<0
$$

In our problem, direct but quite tedious calculations show that on a singular arc, $\frac{\partial}{\partial C} \frac{d^{2}}{d t^{2}} \frac{\partial H}{\partial C}=$ $-2 A \alpha^{2} s^{2}$, thus every singular arc satisfies the Legendre-Clebsch condition, and for every $(s, r)$. In the following, treatments that contain such a singular arc will be called biologically optimal treatments (BOD). 


\section{Comparison of numerical results}

Theorem 4 states the values that an optimal treatment $C$ for cost $L_{2}$ can take at each time $t$. Using this information, we want to numerically answer Problem 2

To simplify the numerical optimization and get it closer to a medical treatment, some conditions are added. To help the patient recover after a large dose of drug, any period of MTD treatment $\left(C(t)=C_{\max }\right)$ must be followed by a period without treatment $(C(t)=0)$, so that the patient's organism can have a rest. Also, the treatment is given by cycles, during a period of one month (30 days), as it is performed in clinical tests. The numerical optimization is performed on the length of each time arc, using built-in optimization tools from Scilab. We chose $B / A=m^{2}$ for size coherence.

During some tested treatments, long periods without or with little treatment make the resistant population decrease a lot. Numerical errors might then let $r<0$ on such periods. To prevent this effect, we include a reserve of quiescent resistant cells: a constant amount of resistant cells $q:=10^{-3}$ that can produce resistant cells $r$. The equation on $r$ now becomes:

$$
\frac{d r}{d t}=\rho r\left(1-\frac{s+m r}{K}\right)-\beta s r+q .
$$

The results presented on FIG.6c show how the number of cells is kept at a low level for the whole experiment. Periods without treatment allow the sensitive population to gain strength, until they are enough to kill the resistant cells. Then the therapy begins at a low level, maintaining the cells at a certain population number. When resistant cells are almost killed off, massive therapy begins to swipe the remaining sensitive cells. Then the resting time allows the sensitive cells population to recover again.

In order to compare this schedule to others, we determined three different protocols for the same problem. First, we used a numerical optimization software, AMPL, to solve Problem2. Time is discretized with steps of $1 h$. Its results are presented on FIG.6a we will later refer to this protocol as the Fixed Time Optimal (FTO). Second, we implemented the stabilization strategy we defined in 3.1 the results are presented on FIG $6 \mathrm{~b}$ Finally, to illustrate the advantage of metronomic treatments over the ones currently in use in medical protocols, we optimized the cost $L$ for cycling treatments that only allow $C(t)=0$ or $C(t)=C_{\max }$. It is represented on FIG 6d Several interesting informations on those different protocols are summed up in TAB 2

The cost $L$ we chose is minimized by the FTO treatment. The BOD protocol, which includes forced cycles, fares also well under this criterion, while MTD and adaptive protocols present a high cost. The four of them use roughly the same amount of drug during the experiment, but the adaptive protocol is the only one to never use the maximal dosage of $5 n M / h$. For a clinical use, the time with small tumoral charge will be important too: the adaptive protocol does not provide any, while during the two cycling schedules, the Petri dish is regularly ridden of almost all cancerous cells. In the FTO protocol, at the end of the experiment the resistant population is no longer under control: thus, contrary to the other strategies, the mean resistant cells proportion in the tumour at the end is really higher for this situation than the other. Especially, because the aim of the adaptive strategy is to reach a limit population with no resistant cells, it fares way better than the other three protocols on this criterion.

The uses and drawbacks of those different protocols will be discussed in 5

\section{Discussion}

We developed a mathematical model to predict the behaviour of competing cancerous cells under various treatment schedules. A study of the dynamical system gives then an idea of an interesting 

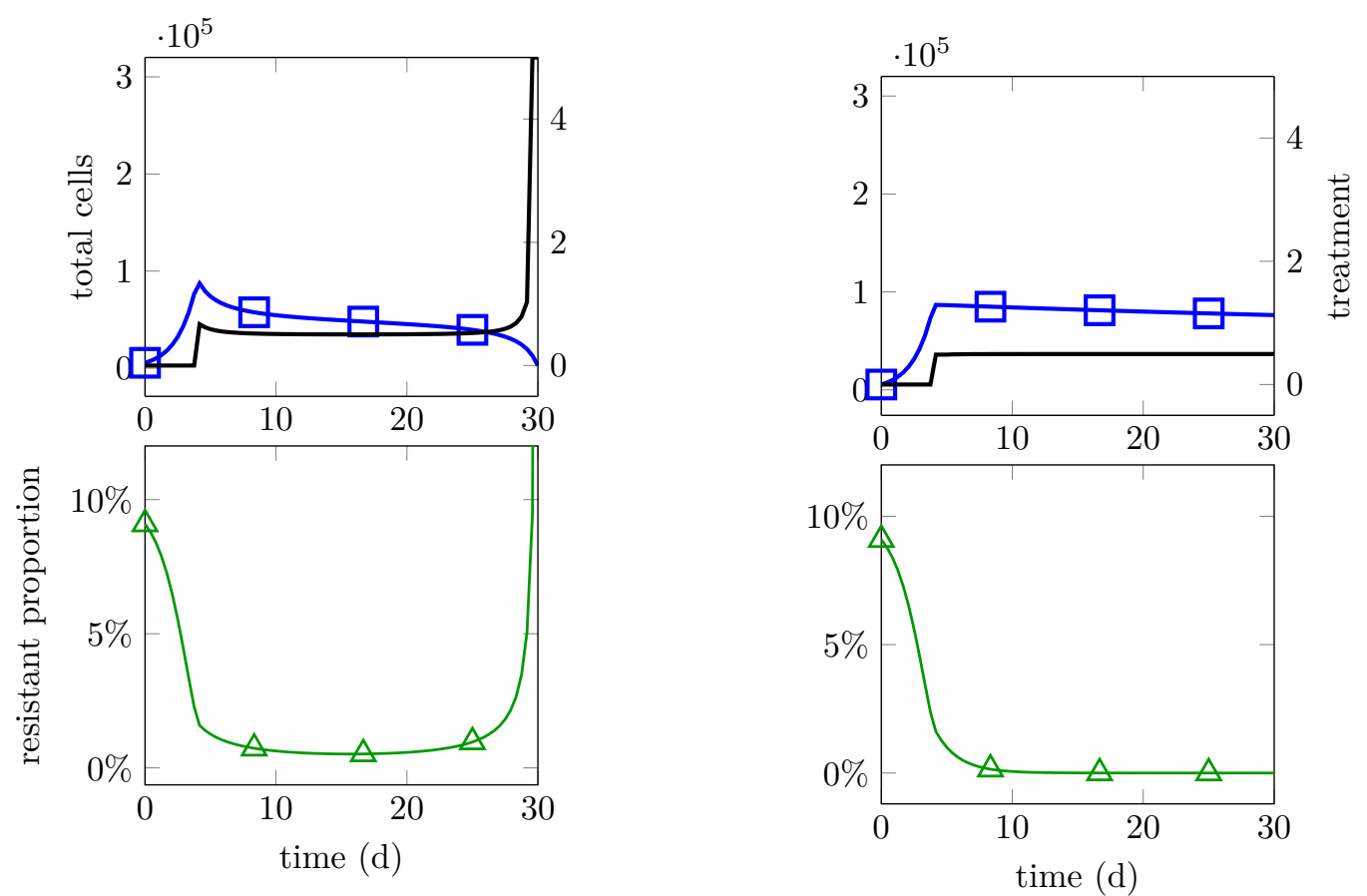

(a) Numerical optimal treatment: Fixed time optimum
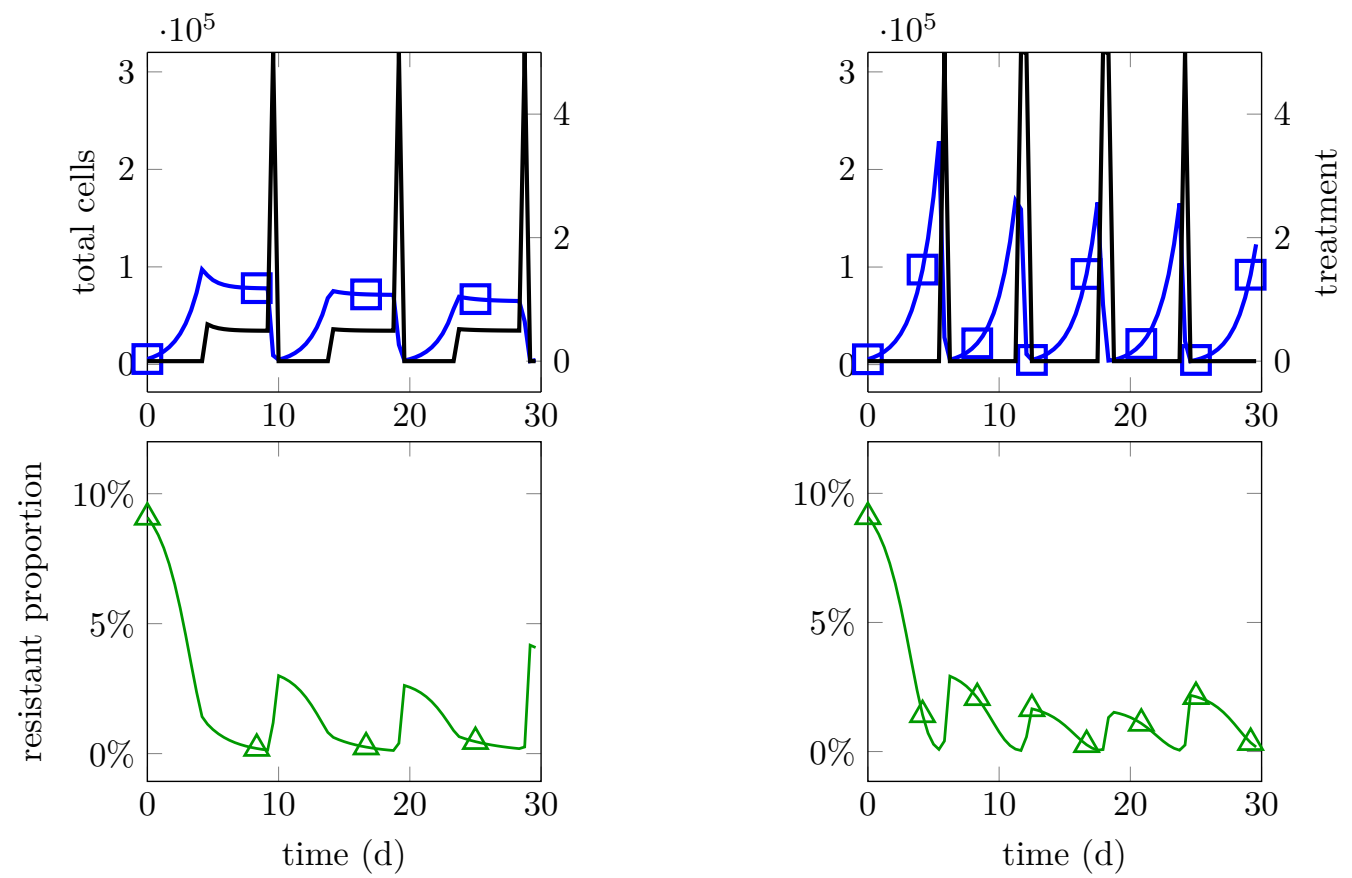

(c) Biologically optimal dose protocol

(d) Maximal tolerated dose protocol

\section{$\square$ total cells $\triangle$ resistant proportion — treatment}

Figure 6: Four different protocols are presented, with the total number of cells in the well coloured in red, the treatment evolution in black, and the resistant cells proportion in green 


\begin{tabular}{|c|c|c|c|c|c|}
\hline & FTO & Adaptive & BOD & MTD & Unit \\
\hline Initial number of sensitive/resistant cells & $5000 / 500$ & $5000 / 500$ & $5000 / 500$ & $5000 / 500$ & cells \\
\hline Total cost $L$ & 1.2 & 2.9 & 1.5 & 3.0 & cells $^{2}$ \\
\hline Maximal cells number & 90700 & 86900 & 97400 & 228700 & cells \\
\hline Total used drug & 380 & 310 & 320 & 300 & $\mathrm{nM}$ \\
\hline Cycles number & $\times$ & $\times$ & 3 & 5 & \\
\hline Total time under $C=C_{\text {max }}$ & 48 & 0 & 72 & 144 & $\mathrm{~h}$ \\
\hline Total time with less than 5000 cells & 24 & 0 & 96 & 144 & $\mathrm{~h}$ \\
\hline Mean percentage of resistant cells the ten last days & 3.98 & 0.0001 & 1.25 & 1.45 & $\%$ \\
\hline
\end{tabular}

Table 2: Comparison between FTO, adaptive, BOD and MTD protocols

schedule of the drug, to stabilize the tumour at a low level, and a further optimization gives an other interesting schedule.

The TAв.2 compares the numerical results of the FTO, adaptive, BOD and an optimal MTD protocols. The FTO protocol answers numerically Problem 2 but would not be considered for a medical trial. Indeed, even if the mathematical cost $L$ is minimal for this protocol among the four schedules we study, it does not ensure a control on the tumour over a long time. As we see in FIG,6a, at the end of the experiment, a large dose of drug is released in order to kill a maximum of sensitive cells. Thus, the resistant cells proportion becomes very important: keeping a control over this tumour after the 30 days treatment might prove more difficult.

The MTD protocol is widely used in medical applications: because it regularly kills a large amount of cancer cells, it is expected that in some cases the tumour will be completely eradicated after the treatment. According to our model, although it does provide the patient a long cumulated time with a reduced tumour charge, it still fares bad on other arguments. First, in order to maintain the resistant cells at a low level, the protocol forces us to allow the tumour to reach a large size at each cycle, which can trigger new effects as the vascularization or metastasis creation, or even kill the patient. Second, as we see on FIG.6d, the resistant cells proportion in the tumour is almost always high, meaning we might be losing control on the disease. Moreover, large doses of drug not only harm the cancerous cells but also the immune system, so long periods under $C_{\max }$ and short recovery times should be avoided as they are very toxic.

The adaptive protocol we described in 3.1 does not use maximal drug dose delivery: the maximal dose in this treatment is $C_{\text {metro }}=0.296 n M / h$, which is far from the maximal $5 n M / h$. This really lessens the treatment toxicity. Its design ensures that the proportion of resistant cells is really low, and decreasing, so that the tumour evolution is really controlled. Moreover, our adaptive protocol can be easily processed in vitro, as it even takes into account some of the variability inherent to real experiments. Further studies of its robustness to the parameters and its speed to reach the interesting equilibrium $E_{s, l}$ should be performed.

The BOD protocol we defined in 3.2 gives an interesting intermediate to the last two protocols. Although we chose the cost $L$ quite arbitrarily, the resulting optimal schedule stabilizes the tumour at a small level, and regularly reduces it to a group of less than 5000 cells. The presence of resting periods in the schedule is also a good point for medical purposes.

Thus, our study highlighted four treatment protocols, that have diverse advantages and drawbacks. It would be a task for medical teams to chose between them, or to define new objectives for us to study if possible.

These results, although encouraging, should be taken into perspective with the model we used.

By construction, our model does not cover the cancer cells total extinction. A simple approach 
to address this problem is to consider that when $s$ or $r$ is less than one cell, then the corresponding population went extinct. But that is not a satisfactory behaviour for the resistant population, as we supposed that even when no treatment is applied, resistant cells exist: $r$ should never be extinct in such model. Even worse, a strong drug dose can in practice kill absolutely all sensitive cells, without any chance of rebound. Knowing that, a MTD protocol as in FIG.6d would probably kill all sensitive cells at one of the drug injections, so that we would loose all control over the resistant population. BOD protocol fares a little better, as periods with maximal dose are shorter, but it still creates a risk of total extinction. In that outlook, our adaptive protocol ensures that the tumour is always controlled, for an indefinite time.

This model highly relies on two hypotheses about the phenomenon of resistance. First, that there exists two genuine populations of cells, one strictly sensitive to the drug and one resistant to it, rather than a continuous trait of resistance. Second, that a lineage never evolves from one category to the other. This might not be the case for a different lineage of cancer cells, and especially not for in vivo experiments. However, this biological model already shows that an intermediate dose of treatment might be better to prevent the resistance of a tumour to a certain drug, rather than MTD treatment schedule.

Another limitation of our model is the fact that no spatial terms are taken into account. It might be interesting to do so, as further experiments by M. Carré performed on tumour spheroids showed that the initial repartition of the cells can have an impact on the outcome. Indeed, at the end of experiments where sensitive cells are predominant, remaining resistant cells tend to be found in clusters. In that case, the Optimal Control Theory will be more complex to use, so numerical optimization solvers could become a more interesting strategy. Finally, for the moment, the toxicity of the drug is not taken into account in the cost $L$ : that may be a further work to consider.

Nonetheless, since this model was developed from considerations of simple, in vitro experiment, it has several advantages. First, very few parameters are needed to simulate effectively the behaviour of the system. They have a good biological interpretation, and can easily be measured. Second, in vitro experiment can be conducted easily compared with experiments involving living animals. Our hypothesis will thus be easy to check, and new experiments do not cost much.

Now that our model produced some optimal schedules, it will be interesting to test them in vitro. This will be performed at the $\mathrm{CRO} 2$, and the results will be analysed and commented by both the medical and the mathematical team. Especially, the adaptive strategy is quite easy to test, and is more likely to be chosen by medical teams.

Although this study presents qualitative and quantitative results on optimal treatments for in vitro experiments, no in vivo direct application is considered: cancerous cells tend to act very differently inside real organs than in a Petri dish, as too many biological phenomenons are set aside in the latter. Our results can be used as guidelines, or hindsight for further experiments, but not as an actual treatment schedule.

\section{Acknowledgements}

I thank Hasnaa Zidani for the numerous discussions we had on optimal control theory and for her help in understanding and manipulating AMPL. I thank Manon Carré for her explanations on cancerous cells interactions, and for having the courage to test our theoretical protocols on in vitro tumours. This work has been carried out in the framework of the project NONLOCAL (ANR-14-CE25-0013), funded by the French National Research Agency (ANR). 


\section{References}

[1] N. André, D. Barbolosi, F. Billy, G. Chapuisat, F. Hubert, E. Grenier, and A. Rovini. Mathematical model of cancer growth controled by metronomic chemotherapies. In $C A N U M$ 2012, 41e Congrès National d'Analyse Numérique, volume 41 of ESAIM Proc., pages 77-94. EDP Sci., Les Ulis, 2013.

[2] David Basanta, Haralambos Hatzikirou, and Andreas Deutsch. Studying the emergence of invasiveness in tumours using game theory. The European Physical Journal B, 63(3):393397, 2008.

[3] Sebastien Benzekry and Philip Hahnfeldt. Maximum tolerated dose versus metronomic scheduling in the treatment of metastatic cancers. Journal of theoretical biology, 335:235$244,2013$.

[4] BG Birkhead and WM Gregory. A mathematical model of the effects of drug resistance in cancer chemotherapy. Mathematical biosciences, 72(1):59-69, 1984.

[5] Brian G Birkhead, Elaine M Rankin, Stephen Gallivan, Leanne Dones, and Robert D Rubens. A mathematical model of the development of drug resistant to cancer chemotherapy. European Journal of Cancer and Clinical Oncology, 23(9):1421-1427, 1987.

[6] Bernard Bonnard and Monique Chyba. Singular trajectories and their role in control theory, volume 40 of Mathématiques \& Applications (Berlin) [Mathematics 86 Applications]. Springer-Verlag, Berlin, 2003.

[7] Alberto Bressan and Benedetto Piccoli. Introduction to the mathematical theory of control, volume 2 of AIMS Series on Applied Mathematics. American Institute of Mathematical Sciences (AIMS), Springfield, MO, 2007.

[8] A. J. Coldman and J. H. Goldie. A stochastic model for the origin and treatment of tumors containing drug-resistant cells. Bull. Math. Biol., 48(3-4):279-292, 1986. Simulation in cancer research (Durham, N.C., 1986).

[9] Alberto d'Onofrio, Urszula Ledzewicz, Helmut Maurer, and Heinz Schättler. On optimal delivery of combination therapy for tumors. Math. Biosci., 222(1):13-26, 2009.

[10] Pedro M Enriquez-Navas, Yoonseok Kam, Tuhin Das, Sabrina Hassan, Ariosto Silva, Parastou Foroutan, Epifanio Ruiz, Gary Martinez, Susan Minton, Robert J Gillies, et al. Exploiting evolutionary principles to prolong tumor control in preclinical models of breast cancer. Science translational medicine, 8(327):327ra24-327ra24, 2016.

[11] Robert A Gatenby, Ariosto S Silva, Robert J Gillies, and B Roy Frieden. Adaptive therapy. Cancer research, 69(11):4894-4903, 2009.

[12] Robert A Gatenby and Thomas L Vincent. Application of quantitative models from population biology and evolutionary game theory to tumor therapeutic strategies. Molecular cancer therapeutics, 2(9):919-927, 2003.

[13] Philip Hahnfeldt, Judah Folkman, and Lynn Hlatky. Minimizing long-term tumor burden: The logic for metronomic chemotherapeutic dosing and its antiangiogenic basis. J. theor. Biol., 220:545-554, 2003. 
[14] Urszula Ledzewicz and Heinz Schättler. Drug resistance in cancer chemotherapy as an optimal control problem. Discrete Contin. Dyn. Syst. Ser. B, 6(1):129-150, 2006.

[15] Urszula Ledzewicz and Heinz Schättler. An optimal control approach to cancer chemotherapy with tumor-immune system interactions. In Mathematical models of tumor-immune system dynamics, volume 107 of Springer Proc. Math. Stat., pages 157-196. Springer, New York, 2014.

[16] Alexander Lorz, Tommaso Lorenzi, Jean Clairambault, Alexandre Escargueil, and Benoît Perthame. Modeling the effects of space structure and combination therapies on phenotypic heterogeneity and drug resistance in solid tumors. Bull. Math. Biol., 77(1):1-22, 2015.

[17] L Marcu, E Bezak, I Olver, and T Van Doorn. Tumour resistance to cisplatin: a modelling approach. Physics in medicine and biology, 50(1):93, 2004.

[18] S. Michelson, B. E. Miller, A. S. Glicksman, and J. T. Leith. Tumor micro-ecology and competitive interactions. J. Theoret. Biol., 128(2):233-246, 1987.

[19] John Carl Panetta. A mathematical model of periodically pulsed chemotherapy: tumor recurrence and metastasis in a competitive environment. Bulletin of mathematical Biology, 58(3):425-447, 1996.

[20] Ariosto S Silva and Robert A Gatenby. A theoretical quantitative model for evolution of cancer chemotherapy resistance. Biology direct, 5(1):1, 2010.

[21] Emmanuel Trélat. Contrôle optimal. Mathématiques Concrètes. [Concrete Mathematics]. Vuibert, Paris, 2005. Théorie \& applications. [Theory and applications]. 